\title{
Clan Mentality: Evidence That the Medial Prefrontal Cortex Responds to Close Others
}

\author{
Fenna M. Krienen, ${ }^{1,2,3}$ Pei-Chi Tu, ${ }^{4,5}$ and Randy L. Buckner ${ }^{1,2,3,4}$ \\ ${ }^{1}$ Department of Psychology and Center for Brain Science and ${ }^{2}$ Howard Hughes Medical Institute, Harvard University, Cambridge, Massachusetts 02138, \\ ${ }^{3}$ Athinoula A. Martinos Center for Biomedical Imaging, Charlestown, Massachusetts 02129, ${ }^{4}$ Departments of Psychiatry and Radiology, Massachusetts \\ General Hospital, Charlestown, Massachusetts 02129, and ${ }^{5}$ Institute of Neuroscience, School of Life Sciences, National Yang-Ming University, Taipei 112, \\ Taiwan
}

Kinship, friendship alliances, and perceptions of others' beliefs guide social interactions and are central to cohesive group behavior. Under certain conditions, brain systems that involve regions along the frontal midline increase activity when inferences are drawn about others who share a similar view to one's own (similarity). A prominent hypothesis is that these regions contribute to social cognition by simulating the other person's perspective based on one's own experience. An alternative is that certain regions process the social relevance of the person (closeness) to oneself and contribute to the assessment akin to signals that govern behavioral approach responses. These alternatives were explored across four functional magnetic resonance imaging experiments $(n=98)$. Experiment 1 localized the target midline regions in the rostral anterior cingulate cortex and anterior medial prefrontal cortex by having participants make personal judgments. The two dimensions (similarity, closeness) were crossed in experiment 2 using actual friends of the participant and unknown others. Making judgments about oneself and friends resulted in increased midline response relative to unknown others regardless of whether the friends shared similar views as the participant. Experiment 3 revealed that similarity was not a factor even when close others were not included. Experiment 4 directly contrasted two extremes: participants made inferences about similar, unknown others and dissimilar friends. Judgments about the close others again increased blood oxygenation level-dependent response along the frontal midline. These results encourage further exploration of the idea that frontal systems linked with limbic circuits facilitate assessment of the relevance or personal significance in social contexts.

\section{Introduction}

The social world of humans is complex and, correspondingly, places great processing demands on the brain. Human, nonhuman primate, and rodent lesion studies suggest that regions along the frontal midline including the anterior medial prefrontal cortex (aMPFC) and rostral anterior cingulate cortex (rACC) may be critical for certain components of social interpretation and behavioral interactions (Glees et al., 1950; Eslinger and Damasio, 1985; Damasio et al., 1990; Rolls et al., 1994; Stone et al., 1998; Anderson et al., 1999; Hadland et al., 2003; Mah et al., 2005; Rudebeck et al., 2006, 2007) [for review, see Damasio et al. (1996), Stuss (2002), and Adolphs (2003, 2009)]. Patients with prefrontal damage, especially in the medial and orbital regions, often lack awareness of social norms and the consequences of

Received April 21, 2010; revised July 29, 2010; accepted Aug. 9, 2010.

This work was supported by the National Institute on Aging (Grants AG-021910 and AG-034556), the Howard Hughes Medical Institute, the Simons Foundation, fellowships from the Department of Defense, and an Ashford Graduate Fellowship in the Sciences (F.M.K.). We thank Jason Mitchell, Jessica Andrews-Hanna, Amitai Shenhav, and Aaron Bornstein for insightful comments and discussion; Dost Ongür and Joel Price for discussion of anatomy; Rebecca Saxe for pointers to relevant literature; and Angela Castellanos, Katherine Powers, and Tammy Moran for assistance with data collection. MRI imaging support was provided by the Harvard Center for Brain Science Neuroimaging Core and the Athinoula A. Martinos Center for Biomedical Imaging.

This article is freely available online through the J Neurosci Open Choice option.

Correspondence should be addressed to Fenna M. Krienen, Center for Brain Science 280.04, Harvard University, 52 0xford Street, Cambridge, MA 02138. E-mail: krienen@wjh.harvard.edu.

DOI:10.1523/JNEUROSCI.2180-10.2010

Copyright $\odot 2010$ the authors $\quad 0270-6474 / 10 / 3013906-10 \$ 15.00 / 0$ their own actions. Neuroimaging studies in normal participants frequently report that self-relevant stimuli result in heightened responses in the MPFC relative to nonsocial stimuli and other control conditions (Amodio and Frith, 2006).

These collective results could suggest that specific regions along the frontal midline contribute to the representation of a core concept of the "self" (Heatherton et al., 2006). By one view, brain systems involving MPFC regions may enable representation of the self and use of that representation to guide decisions about similar others (Mitchell et al., 2006). An alternative possibility is that social cognition expanded or co-opted ancestral brain systems important for processing information that is particularly salient or relevant for future behavior (Damasio et al., 1991, 1996). By this view, the frontal midline may include regions that signal personal significance or respond to a greater degree when personally significant information is available. Social objects, for example, close others including friends and relatives, may be particularly salient stimuli that elicit these signals. Such a process could facilitate social interaction by providing a marker to denote individuals that deserve privileged status in social decisions. Under normal circumstances, personally familiar or relevant individuals are also likely to elicit affective responses or trigger relevant past experiences that may contribute to how individuals are evaluated (Bechara et al., 2003).

Several neuroimaging studies have explored factors that modulate responses within the frontal midline by manipulating the 
perceived similarity of others to the self (Mitchell et al., 2006; Rilling et al., 2008; Mobbs et al., 2009; Volz et al., 2009). Other studies have investigated the effects of closeness or familiarity of the target to the participant (Gobbini et al., 2004; Lou et al., 2004; Schmitz et al., 2004; Ochsner et al., 2005; Heatherton et al., 2006; Zhu et al., 2007; Vanderwal et al., 2008). There are intuitive psychological and evolutionary arguments for the idea that both of these dimensions, similarity and closeness, should be processed by brain systems in the service of social decisions, for instance in aid of identifying social status and processing kin or in-group relationships (Platek and Kemp, 2009).

Here, we explore the relative contributions of similarity and closeness when making social judgments. Foreshadowing the main results, we found that brain regions that respond preferentially during self conditions also respond preferentially when decisions are made about close others, whereas the dimension of similarity neither carried an effect nor interacted with the closeness dimension.

\section{Materials and Methods}

Participants. Ninety-eight young adults participated for payment (mean age $=20.7 \pm 2.1$, 34 male, 64 female). All had normal or corrected-tonormal vision, were right-handed native English speakers, and had no reported history of a neurologic or psychiatric condition. Participants gave written informed consent in accordance with guidelines set by the institutional review boards of Partners Healthcare (Boston, MA) and Harvard University.

Data acquisition. Scanning was conducted on two identical 3T TimTrio scanners (Siemens) at two different sites: the Athinoula A. Martinos Center of Massachusetts General Hospital and the Center for Brain Science at Harvard University. Twelve-channel phased-array head coils were used. Experiment 1 used the following functional imaging acquisition parameters: a gradient-echo echo-planar (EPI) sequence sensitive to blood oxygenation level-dependent (BOLD) contrast [time repetition $(\mathrm{TR})=2500 \mathrm{~ms}$; time echo $(\mathrm{TE})=30 \mathrm{~ms}$; flip angle $(\mathrm{FA})=80^{\circ} ; 3 \times 3 \times$ $3 \mathrm{~mm}$ voxels; field of view $(\mathrm{FOV})=256 \times 256$; interleaved acquisition; 39 slices]. The parameters for functional EPI BOLD runs for experiments $2-4$ were as follows: $\mathrm{TR}=2500 \mathrm{~ms} ; \mathrm{TE}=30 \mathrm{~ms} ; \mathrm{FA}=90^{\circ} ; 3 \times 3 \times 3 \mathrm{~mm}$ voxels; $0.5 \mathrm{~mm}$ gap between slices; FOV $=256 \times 256$; interleaved acquisition; 36 slices. Coverage of the cerebral cortex for all experiments was achieved with slices aligned to the anterior-posterior commissure plane. This slice alignment yielded excellent coverage of most of the frontal cortex with the notable exception of the orbitofrontal cortex (see supplemental Fig. 1, available at www.jneurosci.org as supplemental material).

For all experiments, structural data included a high-resolution T1weighted magnetization-prepared gradient-echo image $(\mathrm{TR}=2530 \mathrm{~ms}$; $\mathrm{TE}=3.44 \mathrm{~ms} ; \mathrm{FA}=7^{\circ} ; 1.0 \mathrm{~mm}$ isotropic voxels; FOV $=256 \times 256$ ). Head motion was minimized by using a pillow and padded clamps, and earplugs were used to attenuate noise. Stimuli were generated on an Apple MacBook Pro (Apple Computer) using MATLAB software (The MathWorks) and the Psychophysics Toolbox extension (Brainard, 1997) and projected onto a screen at the head of the magnet bore. Participants viewed the screen through a mirror attached to the head coil.

Stimulus materials and task procedures. Four separate experiments were conducted using independent samples. In experiment $1(n=31)$ participants were scanned as they performed an attribute-judgment task used in previous studies (Kelley et al., 2002) that included themselves and former U.S. President George W. Bush as targets. Specifically, they were asked to use a four-point scale ( $1=$ not at all; $4=$ quite a bit $)$ to indicate how well single adjectives described the target. Participants responded by using a button box held in their left hands. Trials were presented for $5 \mathrm{~s}$ : stimulus presentation for $4 \mathrm{~s}$ followed by a jittered intertrial interval consisting of a central fixation crosshair that lasted between 1 and $13.5 \mathrm{~s}$ with an average of $3 \mathrm{~s}$. Self trials and Bush trials were ordered using optseq2, an optimization program for scheduling events in event-related experiments (http://surfer.nmr.mgh.harvard.edu/optseq). The stimulus set consisted of 160 single-trait adjectives selected from a pool of normal- ized personality adjectives (Anderson, 1968) and either the word "self" or "Bush" in black text against a white background. Assignment of adjective to condition was counterbalanced across participants. The response scale was present on each trial.

In experiment 2 , participants $(n=28)$ performed a judgment task used in previous studies (Mitchell et al., 2006) in which they were asked to predict how different targets would respond to statements regarding personal preferences. Targets consisted of the participant themselves as well as two personally familiar others (friends) and two personally unfamiliar others (strangers). In addition to this dimension, referred to as closeness to the self, the targets also varied on the dimension of similarity. This was achieved in the following manner: $2-5 \mathrm{~d}$ before the scanning session, participants chose one friend they perceived to be similar to themselves and one that they perceived to be dissimilar to themselves. They were required to choose friends who they knew very well and were of the participant's own sex. Family members and significant others could not be selected. Participants then filled out an online battery of personality, identity, and demographic questionnaires once each for the self and the two friends. Based on these materials we then constructed, for every participant individually, brief fictitious biographies of two strangers. The biographies were short paragraphs containing demographic, personality, lifestyle, and occupational/educational information. Critically, in each case the similar and dissimilar strangers were constructed to be only as similar and dissimilar to the self as were the participants' self-reported assessments of their friends along the dimensions included in the biographies. For example, if a participant is a self-described liberal Democrat and indicates that his similar friend is a moderate Democrat and his dissimilar friend is a moderate Republican, the similar and dissimilar strangers would also be a moderate Democrat and Republican, respectively. In this manner, biographical details for the strangers were tailored to match those provided by the participant for the friends, not for exact content but rather for the degree of distance from the self on each dimension. Care was taken to avoid constructing biographies that depicted either stranger as a common stereotype.

Participants provided a casual headshot of themselves and their two friends before the scanning session. Photos were converted to grayscale images and cropped to include only the face and then identically resized. The photos were used as stimuli in the scanning session task. Additionally, counterbalancing of the photos was achieved in the following manner: the photos of the similar and dissimilar friends of each participant were used as the photos for the fictitious strangers for another participant, crossing similarity factor such that the similar friend photo of participant $n$ became the dissimilar stranger photo for participant $n+1$. We verified with each participant that they did not know or recognize the strangers before proceeding with the scan.

Just before the scanning session, the task was explained and participants were given the photographs and biographies of the two strangers to view. It was emphasized that memorization of the biographies was not necessary because none of the specific details would appear in the scanning session task. The task was to make judgments about personal preferences for each of the five targets. Each trial consisted of a target photo (self, similar friend, dissimilar friend, similar stranger, dissimilar stranger), a short statement about a personal preference (e.g., prefers window seats to aisle seats when flying), and a four-point scale $(1=$ not at all to $4=$ a great deal). Participants had $7.5 \mathrm{~s}$ to indicate their response by using four buttons on a button box with their right hands. They were encouraged to think fully about the most appropriate response, but were instructed to make the button press as soon as they had come to a conclusion.

The task consisted of 100 unique preference statements, amounting to 20 trials per condition. Participants completed the task in four runs each containing 25 trials that were interleaved with variable-duration fixation events to optimize jitter (Dale, 1999; Miezin et al., 2000). The runs lasted $5 \mathrm{~min}, 12 \mathrm{~s}$ each. The assignment of each statement to a target condition and run order were counterbalanced across participants. After the judgment task participants completed $\sim 50$ min of resting-state scan conditions as reported elsewhere (Van Dijk et al., 2010).

A self-paced postscan questionnaire, consisting of questions that probed participants about their experience of each trial during the scan, was administered for 26 of the 28 participants. Specifically, they were 
shown all 100 trials again and asked to respond on a seven-point scale ( $1=$ not at all to $7=$ a great deal $)$ how much they endorsed the following statements about each trial: "I recalled a specific memory or anecdote," "I responded based on what I would have done," "I responded based on their personality," and "I experienced a vivid mental image." After the postscan questionnaire, participants were asked to rate how similar each target was to all of the other targets on a scale of 1-7 ( $1=$ least similar to $7=$ most similar).

The experimental stimuli and procedure for experiments 3 and 4 were largely similar to those of experiment 2 . In experiment $3(n=22)$, participants completed the same scanning task as in experiment 2 , but only the self, similar stranger, and dissimilar stranger were included as targets. One participant was excluded because of excessive movement during the scanning session, leaving 21 participants in the final analysis. Participants completed the prescan online battery using only the self as a target. In this experiment, the strangers' biographies were constructed by recombining the biographical details previously generated for the stranger conditions in experiment 2 and matching them to the online questionnaire data individually for each participant. As previous studies manipulating similarity to self (Mitchell et al., 2006) have focused on the political orientation of self and others, we additionally implemented the constraint that the similar and dissimilar Stranger should have a similar and dissimilar political orientation, respectively, to the participant. The photos of the friends in experiment 2 were used as the stranger photos in experiment 3 . Participants received 32 unique trials per condition (self, similar stranger, dissimilar stranger), arranged using optseq 2 across four runs of 24 trials each. Instead of completing the postscan strategy questionnaire as participants did in experiment 2 , after the scanning session these participants were shown all of the trials once again and asked to respond using only themselves as targets. We repeated the final similarity rating question used in experiment 2 , and additionally the participants were asked to indicate on a seven-point scale how much they liked each target. They were also asked to indicate how likeable they considered themselves to be to others.

In experiment $4(n=17)$ the targets were the self, a similar stranger, and a dissimilar friend. Participants were asked to select only one friend that they knew well and perceived to be dissimilar to themselves. We explicitly required participants to select a friend whose political orientation differed from their own. They then provided headshots of themselves and their friend and completed the online prescan questionnaire battery for both targets. Other participants' dissimilar friend photos served as similar other photos in the same counterbalancing procedure used in experiment 2 . The task materials and procedure were otherwise identical to those used in experiment 3.

Data preprocessing. Preprocessing of the functional magnetic resonance imaging (fMRI) task data included 1) removing the first four volumes to allow T1 equilibration to occur, 2) compensation of systematic, slice-dependent time shifts, and 3) motion correction within and across runs. Data were spatially normalized to the Montreal Neurological Institute (MNI) standard anatomical atlas space (2 $\mathrm{mm}$ isotropic voxels) using a T2-weighted EPI BOLD-contrast atlas in SPM2 (Wellcome Department of Imaging Neuroscience, London, UK) (Friston et al., 1995). Spatial smoothing was achieved with a $6 \mathrm{~mm}$ full-width half-maximum Gaussian kernel.

Whole-brain and region of interest analyses. After preprocessing, data were analyzed using the general linear model. Regressors of no interest included session means, low-frequency linear trends accounting for scanner drift, and six parameters obtained by correction for rigid body head motion. For each participant, the BOLD response to each trial type (i.e., in experiment 1: self or Bush) was estimated using a canonical hemodynamic response function along with its temporal derivative. Events were time-locked to stimulus onset. Contrasts of interest were constructed, and whole-brain activation maps were generated by converting the $t$ statistics to $z$ statistics.

Hypothesis-driven region of interest (ROI) analyses were performed in the following manner: a ROI with radius of $8 \mathrm{~mm}$ was drawn around the site of maximally increased BOLD response in the frontal midline from the contrast self $>$ Bush in experiment 1 . This ROI was within the rACC centered at coordinates $x=-4, y=34, z=0$ in the MNI atlas.
Although rACC has often been observed in prior studies that survey similar contrasts, it is embedded within a larger response along the frontal midline that extends into other regions of the MPFC. Meta-analyses have emphasized the importance of the aMPFC to aspects of social cognition that involve mentalizing and self-referential processing (e.g., Amodio and Frith, 2006). For this reason we additionally selected a ROI within the aMPFC around a second peak from the self $>$ Bush contrast $(x=-4, y=56, z=10)$. We adopt the view of Ongür et al. (2003) that the rACC and aMPFC are anatomically distinct, yet heavily interconnected, components within the MPFC, which itself is distinguished from another frontal system comprised mainly of orbital areas. The two a priori MPFC ROIs were carried forward to investigate the effects of similarity and closeness in experiments $2-4$. As will be demonstrated, within the context of our manipulation the two regions largely showed similar response properties consistent with the view that they participate in a common functional network. We did not select regions within the orbitofrontal cortex but that should not be taken as an indication the region did not respond to our task manipulations. Rather, the loss of signal-tonoise ratio (SNR) owing to susceptibility artifacts (Ojemann et al., 1997) precluded making any observations in the most ventral prefrontal regions (see supplemental Fig. 1, available at www.jneurosci.org as supplemental material).

For experiments 2-4, time courses for each condition were extracted from the ROIs, and peak response magnitude estimates were calculated by subtracting the mean of time points 1 and 8 from the mean of time points 4 and 5 (the peak of the time course minus the ends for normalization purposes). The magnitude estimates were submitted to random effects models, and comparisons were performed by using a two-factor ANOVA in the case of experiment 2 and paired $t$ tests otherwise.

For all experiments, exploratory whole-brain maps were also provided to allow full inspection of the data. For these maps the data were thresholded at $p<0.001$ uncorrected, allowing full visualization of the BOLD response pattern across the different experiments. Data were projected onto the Caret-inflated cortical surface (Van Essen, 2005).

\section{Results}

\section{Behavioral results}

For experiments 2-4, the prescan and postscan questionnaires were assessed to determine whether our strategies for generating similar and dissimilar targets were successful. In the prescan questionnaire in experiment 2, participants answered a personality inventory for themselves and each of their two friends. We computed a difference score on these data by subtracting the response given for each of the friends from the response given for the self for each item. Dissimilar friends had larger absolute difference scores than did similar friends $\left(t_{(27)}=-6.28 ; p<0.001\right)$. In the postscan questionnaire, participants indicated explicitly that they perceived themselves to be significantly more similar to the similar friend and similar stranger than to the dissimilar counterparts $\left(F_{(1,27)}=124.40 ; p<0.001\right)$ (Fig. $\left.1 A\right)$. There was also a significant effect of closeness on this measure: overall, friends were perceived to be more similar to the self than were strangers $\left(F_{(1,27)}=41.25 ; p<0.001\right)$; however, note that the similar stranger was still perceived to be more similar than the dissimilar friend $\left(t_{(27)}=4.19 ; p<0.001\right)$, suggesting that the similarity manipulation was not confounded by closeness. In experiment 3 (Fig. $1 B$ ) and experiment 4 (Fig. 1C), participants again rated the similar target as more similar to the self than the dissimilar target (experiment 3, $p<0.001$; experiment $4, p<0.005$ ).

In experiment 2 we asked participants explicitly whether they had used themselves as a "proxy" when responding to trials about the other targets. In the postscan rating task, participants endorsed the statement "I responded based on what I would have done" significantly more for similar target trials than for dissimilar target trials (similar rating $=4.33$; dissimilar rating $=3.50$; $\left.F_{(1,25)}=33.51 ; p<0.001\right)$ (Fig. $2 A$ ), consistent with previous 

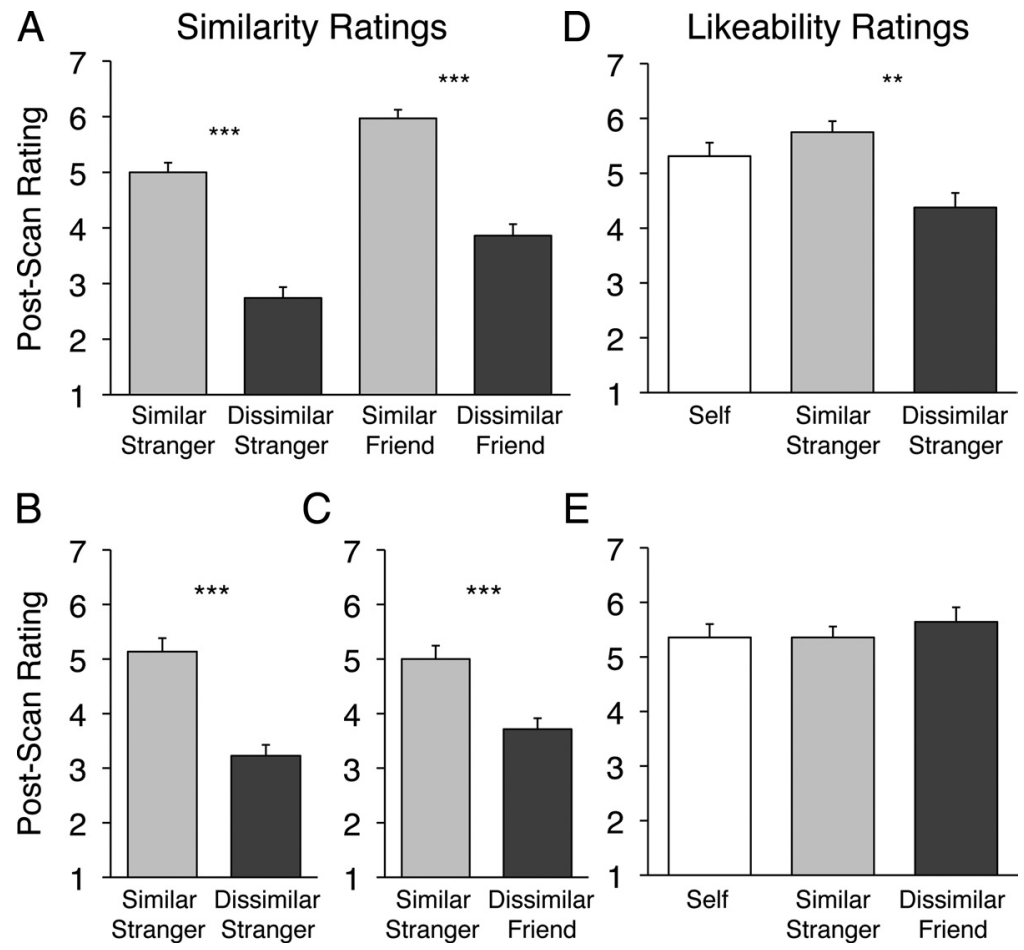

Figure 1. Postscan behavioral ratings of targets in experiments $2-4 . A$, In experiment 2 participants reported perceiving both of the similar targets to be significantly more similar to the self than the respective dissimilar counterparts. $\boldsymbol{B}$, In experiment 3 , the similar stranger was judged to be more similar to the self than was the dissimilar stranger. $\boldsymbol{C}$, In experiment 4 the similar stranger was more similar than the dissimilar friend. $\boldsymbol{D}, \boldsymbol{E}$, Likeability ratings were collected postscan in experiment $3(\boldsymbol{D})$ and experiment $4(\boldsymbol{E})$. $y$-axis scale ranges from 1 to 7 $(1=$ least to $7=$ most $) .{ }^{* *} p<0.01 ;{ }^{* * *} p<0.005$.
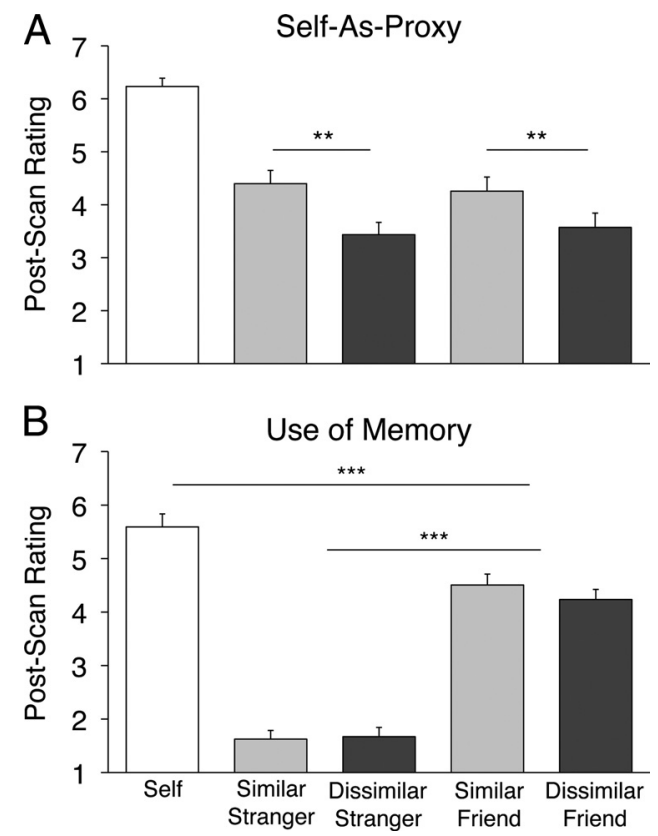

Figure 2. Postscan strategy assessments in experiment 2. A, Participants endorsed the statement "I responded based on what I would have done" significantly more often for similar strangers and friends than for dissimilar targets. There was no effect of closeness on this rating. $\boldsymbol{B}$, Participants endorsed the statement "I relied on a particular memory or anecdote" significantly more for friends than for strangers. $y$-axis scale ranges from 1 to $7(1=$ least to $7=$ most $) .{ }^{* *} p<0.01$; ${ }^{* *} p<0.001$.

work suggesting that the self is perceived to be a more appropriate reference point for reasoning about similar others than for dissimilar others (Cadinu and Rothbart, 1996). There was no effect of closeness $(p>0.9)$, indicating that personal relationship did not play a role in the degree to which participants endorsed this strategy. Participants indicated that they "relied on a particular memory or anecdote" significantly more for friends than for strangers (mean friend rating $=$ 4.37; mean stranger rating $=1.65 ; F_{(1,25)}$ $=11.19 ; p<0.001)$, with no effect of similarity $(p>0.5)$ (Fig. $2 B$ ). They also reported using specific memories more for self trials than for friend trials $\left(_{(25)}=\right.$ $-5.91 ; p<0.001)$.

In experiments 3 and 4 we asked participants to make explicit likeability judgments about the targets. Similar strangers were judged to be more likable than dissimilar strangers in experiment 3 (like similar stranger $=5.69$; like dissimilar stranger $=4.36 ; t_{(20)}=4.39 ; p<0.001$ ) (Fig. $1 D$ ), whereas there was no difference in likeability for the similar stranger and dissimilar friend in experiment 4 (like similar stranger $=5.36$; like dissimilar friend $=5.64 ; t_{(16)}=0.75 ; p>0.50$ ) (Fig. $1 E)$. Therefore, similarity is not uniformly confounded with likeability in our experiments.

We also examined the prescan questionnaires from experiment 2 to determine whether the two friend targets were appropriately matched on a variety of potentially confounding factors related to closeness. Based on the questionnaires, we found no difference in terms of how long the participants had known their two friends (i.e., the duration of the friendship) (know similar friend $=6.01$ years; know dissimilar friend $=5.23$ years; $t_{(27)}=0.75 ; p>0.45$ ) and how often they were in contact, both in terms of face-to-face contact ( similar friend $=2.71 \mathrm{~h} /$ week; dissimilar friend $=3.41$ $\mathrm{h} /$ week; $t_{(27)}=-0.97 ; p>0.30$ ) and other forms of communication such as phone calls and E-mail ( similar friend $=1.52$ $\mathrm{h} /$ week; dissimilar friend $=1.27 \mathrm{~h} /$ week; $t_{(27)}=0.61 ; p>$ $0.50)$. These data points converge to suggest that we successfully manipulated the similarity dimension and participants chose two friends they knew equally well.

During the scanning session participants were significantly faster to respond to friend trials than to stranger trials [friend response time $(\mathrm{RT})=3.83 \mathrm{~s}$; stranger $\mathrm{RT}=4.04 \mathrm{~s} ; F_{(1,27)}=13.37$; $p=0.001$ ] and to self trials faster than stranger trials (self RT $=$ $\left.3.76 \mathrm{~s} ; t_{(27)}=-4.19 ; p<0.001\right)$ in experiment 2 . There was no difference in response time for self and friend trials $(p>0.1)$. There was also no effect of similarity on response time $(p>0.5)$. These response time patterns held for similar comparisons in experiments 3 and 4 . For experiment 3, self RT $=3.44 \mathrm{~s}$; similar stranger RT $=3.79 \mathrm{~s}$; dissimilar stranger RT $=3.74 \mathrm{~s}$; self RT vs stranger RT, $t_{(21)}=-5.32, p<0.001$; similar stranger RT vs dissimilar stranger RT, $p>0.2$. For experiment 4 , self RT $=$ $3.15 \mathrm{~s}$; similar stranger $=3.54 \mathrm{~s}$; dissimilar friend $=3.27 \mathrm{~s}$; self RT vs similar stranger RT, $t_{(16)}=-5.54, p<0.001$; similar stranger vs dissimilar friend, $t_{(16)}=-3.50, p<0.01$. Because the preference statements in the scanned task were unique (not repeated) across conditions, comparisons of the behavioral responses themselves are not informative. 


\section{Cortical responses during self trials} relative to an unknown other

In experiment 1 , whole-brain analysis was performed for the contrast self $>$ Bush. Consistent with previous results, regions significantly more responsive during self trials were prominent within the frontal midline including the rACC and aMPFC. Additional regions of prominent response included the midcingulate cortex, lateral prefrontal cortex, and inferior parietal cortex (Fig. 3).

The self $>$ Bush contrast was used to define a priori regions that were carried forward for all subsequent analyses. Specifically, ROIs were constructed around the peak of activity in the frontal midline from self $>$ Bush with a radius of $8 \mathrm{~mm}$, retaining only those voxels that survived a threshold of 0.001 , uncorrected (Figs. $4 A$, $5 A)$. This procedure identified regions within or near the rACC and aMPFC. We intentionally constrained our ROIs to be relatively small to avoid averaging signal across many brain areas, given the convergence of several different architectonic zones and their corresponding brain networks (Ongür et al., 2003). The constructed rACC ROI is at or near human areas 24a and 32ac (Fig. $4 \mathrm{~A}$ ); the anatomically distinct aMPFC ROI is at or near human area 10 (Fig. $5 A$ ). These two ROIs were carried forward to experiments $2-4$ to allow for unbiased quantification of signal modulation across the range of conditions.

rACC and aMPFC responses dissociate friends from strangers In experiment 2 , self trials resulted in reliably greater BOLD response in $\operatorname{rACC}\left(t_{(27)}=5.56 ; p<0.001\right)$ (Fig. $4 B$ ) and aMPFC $\left(t_{(27)}=5.02 ; p<0.001\right)$ (Fig. $\left.5 B\right)$ relative to stranger trials. Interestingly, self trial responses were also reliably greater than friend trial responses in $\operatorname{rACC}\left(t_{(27)}=3.23, p<0.01\right)($ Fig. $4 B)$, but not in aMPFC $\left(t_{(27)}=0.39 ; p>0.7\right)$ (Fig. $\left.5 B\right)$. This dissociation was the only one observed in the response patterns of our rACC and aMPFC ROIs. Friend trials resulted in significantly increased response in both regions relative to stranger trials

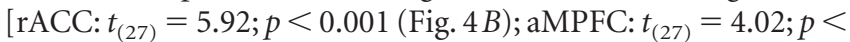
0.001 (Fig. 5B)]. However, there was no main effect of similarity in either region [rACC: $t_{(27)}=0.73 ; p>0.4$ (Fig. $4 B$ ); aMPFC: $t_{(27)}=-0.68 ; p>0.5$ (Fig. $5 B$ ) ] and no interaction, lending to the interpretation that closeness to self but not similarity to self dissociate the responses in both the rACC and aMPFC.

To replicate and extend this result we conducted two additional experiments using different groups of participants. Previous research using a similar paradigm reported differences in the frontal midline response when participants made judgments about similar versus dissimilar others. It is possible that the inclusion of the friends in experiment 2 may have caused participants to adopt a different strategy when reasoning about strangers than they might have in the absence of that condition. Social context can affect perceptions about in-group constituency (Haslam et al., 1992). Thus, to determine whether our results in experiment 2 can be attributed to a context or referencing effect, experiment 3 involved a version of the task that included only self, similar stranger, and dissimilar stranger conditions.
Self $>$ Bush

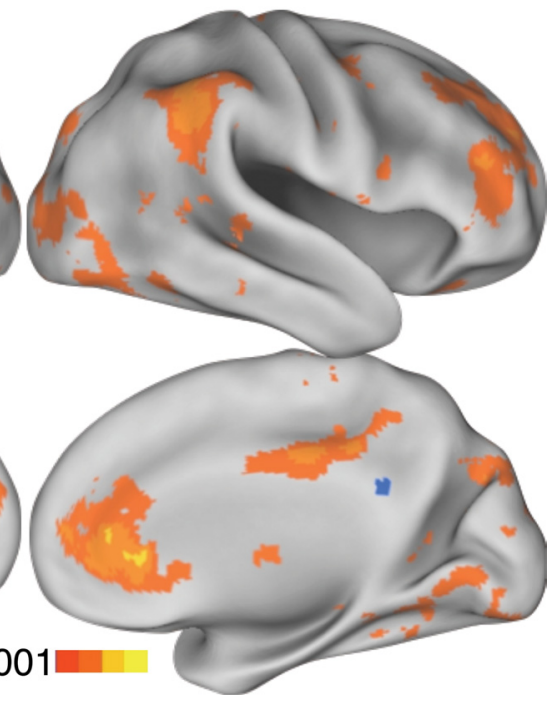

sponse in the frontal midline. In experiment 1 making attribute judgments about the self (warm colors) relative to a well known, but personally unfamiliar, other (former U.S. president George W. includes the $\mathrm{rACC}$ and aMPFC in the frontal midline and the midcingulate cortex. Regions in the rACC and aMPFC modulating in this analyses in experiments $2-4$. Maps are thresholded at $p<0.001$, uncorrected.

Results replicated those of experiment 2: there was no difference between similar stranger and dissimilar stranger trials in either $\operatorname{rACC}\left(t_{(20)}=0.28 ; p>0.7\right)$ (Fig. $4 C$ ) or $\operatorname{aMPFC}\left(t_{(20)}=-0.72\right.$; $p>0.4$ ) (Fig. $5 C$ ), and stranger conditions resulted in significantly decreased BOLD response relative to the self condition in both $\operatorname{rACC}\left(t_{(20)}=-10.20 ; p<0.001\right)$ (Fig. $\left.4 C\right)$ and aMPFC $\left(t_{(20)}=-7.20 ; p<0.001\right)$ (Fig. $5 C$ ).

Experiment 4 included the conditions self, similar stranger, and dissimilar friend. The purpose was to directly test the two extremes of our dimensions. Once again, the dissimilar friend activated rACC $\left(t_{(16)}=3.88 ; p<0.001\right)$ (Fig. $\left.4 D\right)$ and aMPFC $\left(t_{(16)}=2.23 ; p<0.05\right.$ ) (Fig. $\left.5 D\right)$ more than did the similar stranger.

To summarize, in experiments $2-4$ the rACC and aMPFC ROIs exhibited a main effect of friends $>$ strangers and failed to find an effect of similarity (Figs. $4 B-D, 5 B-D$ ). However, it is interesting to note that although the magnitude estimate for self trials was significantly greater than friend trials in the rACC (Fig. $4 B, D$ ), this difference was not observed in the aMPFC ROI (Fig. $5 B, D$ ).

\section{Whole-brain analyses}

The results from the above regional analyses were confirmed in whole-brain comparisons of conditions of interest in experiments 2-4. Figure 6 shows the results of four comparisons in experiment 2 projected onto the inflated cortical surface: self $>$ strangers (Fig. 6A), friends $>$ strangers (Fig. 6B), self $>$ friends (Fig. $6 C$ ), and similar $>$ dissimilar, collapsing across the closeness dimension (Fig. 6D). As can be appreciated visually, the self $>$ strangers and friends $>$ strangers contrasts yield largely overlapping networks of brain regions in the MPFC, posterior cingulate/ retrosplenial cortex, inferior parietal lobe, lateral temporal cortex, and medial temporal lobe (MTL). The contrast self $>$ friends shows that self trials result in relatively higher responses in the rACC and the midcingulate as well as in the insula and temporoparietal junction (TPJ), whereas friends trials activate the ventromedial prefrontal (VMPFC) and the posterior cingulate/precuneus (Fig. 6C). 

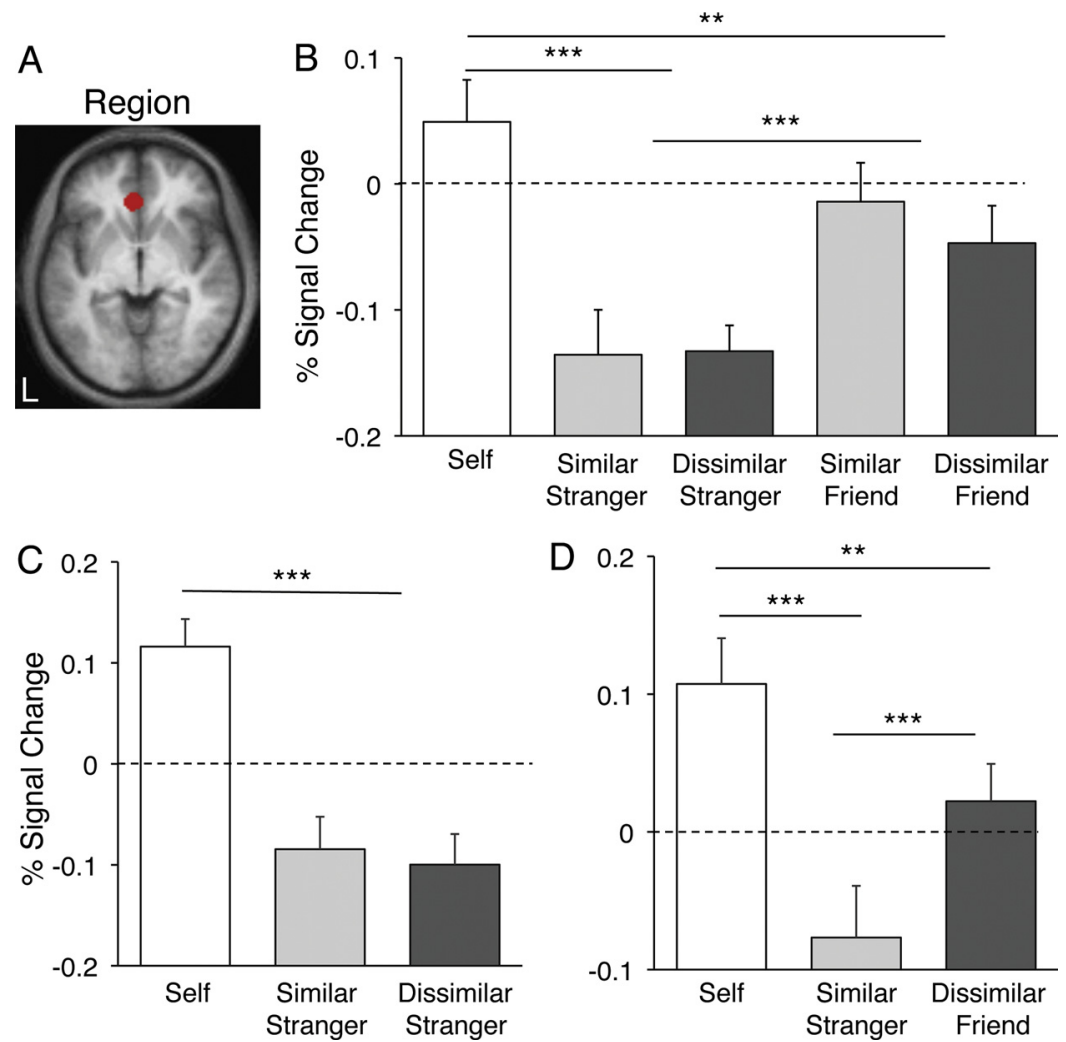

Figure 4. Regional analysis on $\mathrm{rACC}$ distinguishes friends from strangers. $A, A$ ROl of $\sim 8 \mathrm{~mm}$ radius was drawn around the peak in the rACC from the contrast self $>$ Bush in experiment 1 (see Fig. 3). $\boldsymbol{B}$, Parameter estimates for the ROl obtained in experiment 2 indicate that close others (friends) activate this region more than unfamiliar others (strangers), whereas the similarity manipulation produces no effect. $\boldsymbol{C}, \boldsymbol{D}$, Experiment 3 replicated the lack of similarity effect seen in experiment 2, whereas experiment 4 contained only the two extremes of the conditions (similar other, dissimilar friend) to show again that the dimension of closeness, not similarity, appears to drive the response in this region. ${ }^{* *} p<0.01 ;{ }^{* *} p<0.001$.

In contrast to the robust results generated by comparing friends with strangers, the contrast of similar $>$ dissimilar conditions and the reverse contrast failed to produce notable effects across the brain (Fig. 6D).

This pattern of results generalized to these contrasts in experiments 3 and 4 . Self $>$ strangers in experiment 3 again activated the aMPFC and rACC and the TPJ, insula, and MTL (Fig. 7A). Within the similarity dimension, the contrast similar stranger $>$ dissimilar stranger again failed to produce differences at the whole-brain level at the liberal threshold of $p<0.001$ (Fig. 7B). In experiment 4 , too, self $>$ similar stranger again resulted in increased response in MPFC, insula, and MTL (Fig. 7C), Additionally, the pattern of BOLD response during dissimilar friend trials more closely resembled that seen in self trials than it did the similar stranger trials (Fig. 7D), although again the VMPFC (Fig. 6C) was found to be more active for dissimilar friend $>$ self (data not shown).

One important caveat to these whole-brain results is the low SNR in the orbitofrontal cortex (supplemental Fig. 1, available at www.jneurosci.org as supplemental material). For this reason we cannot preclude the possibility that orbitofrontal and other compromised regions also respond differentially to our task conditions.

Because of response time differences between conditions, we repeated the above analyses adding response time on each particular trial as a regressor of no interest. This analysis yielded results similar to those reported above.

\section{Discussion}

Dunbar and Shultz (2007) proposed that a critical event in the evolution of anthropoid species was the emergence of the ability to create and maintain relationships beyond reproductive partners. In humans this ability reaches a remarkable level of sophistication. By the age of 5 or 6 , children can flexibly process social information, represent mental states of others, and engage with peers and family in a way that maintains cohesive group interactions (Flavell, 1999; Wellman et al., 2001; Hughes and Leekam, 2004; Fehr et al., 2008). Here, we explored two aspects of social cognition (similarity and closeness) to better understand the contributions of brain systems supporting social cognition. We focused on the MPFC. Social abilities are compromised by lesions to the frontal midline including the aMPFC and rACC (Damasio et al., 1990; Rolls et al., 1994; Stone et al., 1998; Anderson et al., 1999; Mah et al., 2005), and recent neuroimaging studies of mental state attribution consistently observed that these brain regions respond robustly during social judgments [for review, see Amodio and Frith (2006), Northoff et al. (2006), and Mitchell (2009)].

\section{Implications of MPFC modulation to social closeness}

Similarity and closeness represent two possible facets by which brain systems might process attributes of others. The perception that another person has similar views to oneself provides information that can be used to guide social decisions by allowing one to use one's own behavior as a proxy to predict mental states of the like-minded person. Motivated by this possibility, Mitchell et al. (2006) suggested that increases in MPFC activity when people make judgments about similar others provides evidence that the MPFC contributes to mental simulations during social cognition (see also Buckner et al., 2008 for a related proposal). In this article we explored an alternative explanation for the contribution of regions to social cognition, namely that they evaluate or provide a signal reflecting the personal significance of close others.

There is considerable evidence that social species develop mechanisms to aid in identifying and evaluating kin, in-group members, and individuals otherwise relevant to one's survival (Trivers, 1971; Rendall et al., 1996). Considering social cognition from this perspective, neural mechanisms may have evolved for distinguishing personally significant social information. Across a series of studies we consistently found that regions within the MPFC respond strongly to judgments about personally relevant individuals, exhibiting a dissociated response profile when making judgments about close others (friends) relative to unfamiliar others (strangers).

These results suggest that similarity is not always a dimension that modulates responses in the MPFC. One aspect of our design that may distinguish it from other experiments that report a similarity effect is that we tailored our descriptions to each participant to match the "extremeness" of the strangers' attributes to that of the participant's friends, which produced less extreme individuals than some prior studies (e.g., Mitchell et al., 2006). Harris and Fiske (2007) found that pictures of "socially 
undesirable" people such as the homeless result in more reduced MPFC response than pictures of "socially desirable" people. Given the link between the MPFC and the limbic system (see below), it is not unreasonable to suspect that certain MPFC regions may track the value assessment of an unknown other rather than their perceived similarity to the self.

Although similarity was not found to have an effect in our experiments, affective evaluations do interact with mentalizing processes (Hornak et al., 2003; Moran et al., 2006; Olsson and Ochsner, 2008). Mobbs et al. (2009) recently reported a correlation between likeability and perceived similarity in situations of vicarious reward of personally unknown, but socially desirable, targets. They found that both dimensions correlated with activity in the MPFC, although subtraction of the likeability ratings from the similarity ratings still resulted in significant ACC activity. The response they noted corresponds closely to the rACC and aMPFC regions in our experiments (Fig. 4). We explicitly avoided including traits in the strangers' biographies that could be perceived as strongly desirable or undesirable (traits of both similar and dissimilar strangers were always framed in neutral to positive terms) to provide a clean test of the similarity dimension while controlling for affective valuation. Similar and dissimilar friends were judged to be equally likeable; one interesting future test would be to use close others with whom the participant does not get along very well.

Thus, although perceived similarity may play an important role in social interactions generally (Cadinu and Rothbart, 1996), judgments of others need not necessarily arise from using the self as a reference point (Higgins and Bargh, 1987). This may be particularly true when past experience with others can be brought to bear on current decisions or evaluations. Our results suggest that the rACC and aMPFC regions discussed here dissociates judgments that are self-relevant from those that are not (Moran et al., 2006; Schmitz and Johnson, 2006). Although the concept self-relevance is nebulous, here it would correspond to salient or valued others with whom one has personal involvement.

\section{Does the MPFC contribute specifically to social cognition?}

The view of social cognition as an autonomous set of mental operations distinct from other forms of cognition has long been debated in social psychology (Fiske and Taylor, 1984; Higgens and Bargh, 1987). Neuroscientific data are increasingly being brought to bear on this question (Mitchell, 2009). It is thus important to note that explicitly nonsocial tasks also engage the frontal midline, in particular those that require calculating expected reward or value of a choice (e.g., Daw et al., 2006; see also Beckmann et al., 2009 for meta-analysis). Lesions to human ventromedial PFC result in failure to represent the consequences of choices in nonsocial decision-making tasks (Bechara et al., 1994, 2000).
Whether the representation is selectively social or related to valuation of a stimulus or an outcome more generally is unclear (Adolphs, 2009, 2010; Rushworth et al., 2007; Beckmann et al., 2009). Lesions to anterior cingulate gyrus in the monkey do decrease interest in social stimuli (Rudebeck et al., 2006), but while social information is both rewarding and incontrovertibly valuable (Anderson, 1998), this is especially true when such information is useful for behavior. For instance, thirsty monkeys will forgo juice to view pictures of other monkeys, but only when they are socially relevant (e.g., either of higher status or a possible mate) (Deaner et al., 2005).

Tomlin et al. (2006) reported rACC activity in an economic exchange game during observation of partner decisions that were relevant to the participant, but not during a control condition with personally relevant outcomes that were not generated by a social partner, i.e., by a computer. One interpretation of these results is that rACC activity indexed the social component of the task. Another interpretation is that social interactions, especially in the context of trust games when one's own actions depend on those of another, inherently contain an element of uncertainty. Under this perspective, the rACC codes the behavioral relevance or affective value of a social interaction rather than the social nature of the task per se. Bush et al. (2000), in a comprehensive review, noted that regions of the ACC near to our region respond to a range of tasks laden with affective content, many of which do not have explicit social demands. These analyses do not provide insight into whether all specific MPFC regions show generaliza- 


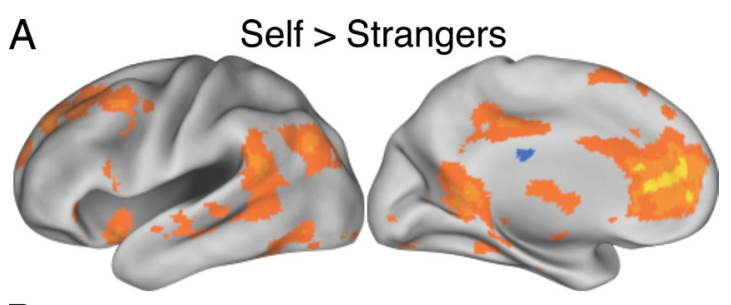

B
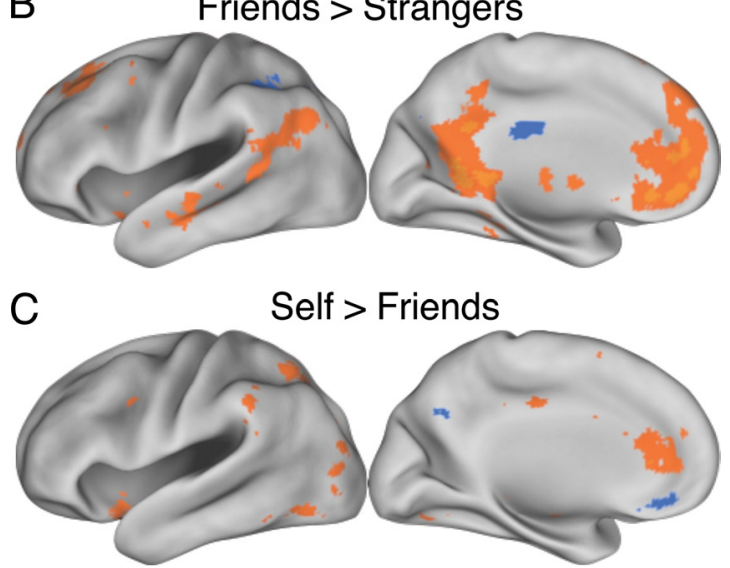

D

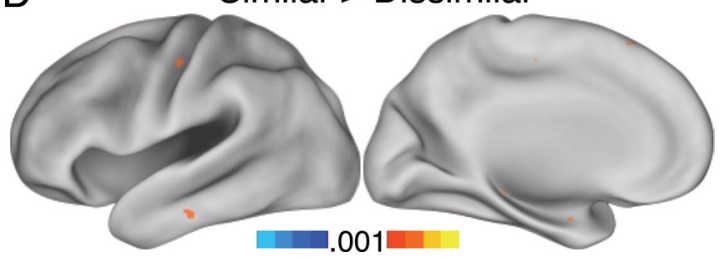

Figure 6. Whole-brain contrasts for experiment 2. $\boldsymbol{A}, \boldsymbol{B}$, Making mental state inferences about the self and friends activates a similar network relative to strangers. $C$, Relative to the self condition, judgments about friends increase response in the MPFC. D, There was no appreciable difference between the similar and dissimilar conditions.

tion to nonsocial contexts, but do suggest that the role of MPFC in social cognition can be profitably understood by considering the more general role of the region and its associated networks in affective processing and valuation.

\section{Anatomical constraints and possible relationship to limbic networks}

Assessing the present observations in relation to monkey anatomy provides further insight into possible functions, although many aspects of relevant anatomy have not been investigated in sufficient detail to draw strong conclusions. Meta-analyses of neuroimaging studies using social content have emphasized the aMPFC (e.g., Amodio and Frith, 2006; Northoff et al., 2006; Mitchell, 2009). Responses falling within the rACC are also frequently observed in studies of social cognition (Mitchell et al., 2002; Heatherton et al., 2006; Moran et al., 2006; Ames et al., 2008, Vanderwal et al., 2008). Based on architectonic evidence in the human (Ongür et al., 2003; Palomero-Gallagher et al., 2008), these two regions are certain to be comprised of multiple, distinct areas. However, estimated homologues in the monkey suggest that they may be components of an interacting network.

Specifically, human aMPFC and rACC may overlap a "medial network" identified in the monkey that is concerned with visceral and affective systems and is largely distinct from an "orbital network" consisting of mainly ventrolateral-orbital structures (Ongür and Price, 2000). Macaque anterior cingulate areas 24a and

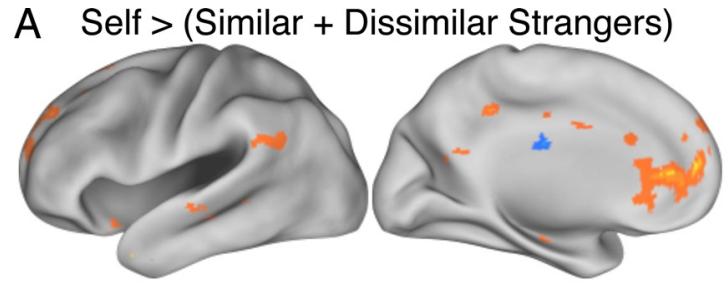

\section{B Similar Stranger $>$ Dissimilar Stranger}
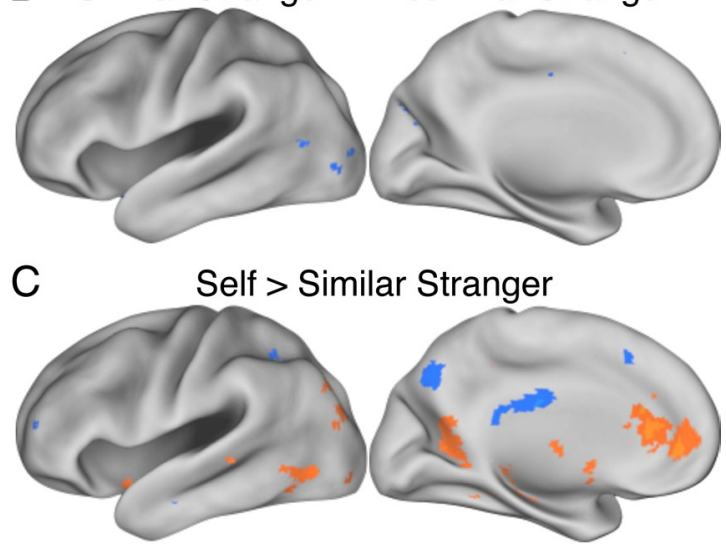

D Dissimilar Friend $>$ Similar Stranger

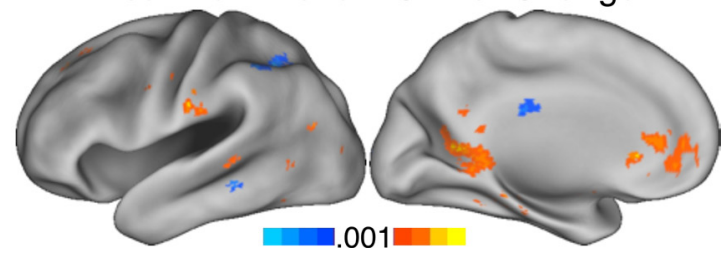

Figure 7. Whole-brain contrasts for experiments 3 and $4 . A, B$, In experiment 3 self $>$ others increases the $\mathrm{rACC}$ and aMPFC response robustly $(\boldsymbol{A})$, whereas little difference appears between similar and dissimilar others $(\boldsymbol{B}) . \boldsymbol{C}, \boldsymbol{D}$, In experiment 4 self $>$ similar stranger replicates experiments 2 and $3(\boldsymbol{C})$, and the contrast dissimilar friend $>$ similar stranger reveals, consistent with experiment 2, loci in MPFC that respond preferentially when making inferences about close others relative to responding about strangers.

caudal 32 are interconnected parts of the medial network that receive few direct sensory inputs but are linked to regions important to viscero-motor function (Carmichael and Price, 1996). In humans, the relative expansion of the frontal pole causes a shift in the locations of areas with areas 10p and 10r falling at or near our aMPFC region (Ongür et al., 2003). Human area 32ac is likely not homologous to area 32 in the macaque (but see PalomeroGallagher et al., 2008). Area 10r, which is adjacent to $32 \mathrm{ac}$ and not identified as a subdivision in the monkey, is architectonically distinct from neighboring cingulate areas and is suspected to have connections to autonomic control systems because its activity tracks visceral arousal measured by electrodermal response (discussed in Ongür et al., 2003).

Analysis of homologies between monkey and human thus suggests constraints on what kinds of information are integrated by the MPFC and also that the broad region, especially area 10, has expanded in recent hominin evolution. Although area 10 is likely heavily interconnected with frontal subdivision comprising a human homolog to the medial network, it probably also directly interacts with limbic structures. Area $10 \mathrm{~m}$ projects to the anterior and posterior cingulate, superior temporal sulcus, amygdale, and hippocampal formation (e.g., Petrides and Pandya, 2007; Saleem et al., 2008). It is reasonable to speculate that a critical processing contribution of the MPFC to social cognition may stem, in part, 
from limbic influences associated with the medial network and direct projections.

Viewing our results from this perspective, one interpretation of "social" tasks such as thinking about close others is that they rely on systems optimized to process information perceived to be significant to the individual. Social judgments may be instances where these systems are maximally used. Evolutionary pressures on social behavior may have caused an expansion of these systems in anthropoid primates, particularly so for humans, such that they are especially adapted to challenges associated with maintaining and navigating social groups.

\section{References}

Adolphs R (2003) Cognitive neuroscience of human social behaviour. Nat Rev Neurosci 4:165-178.

Adolphs R (2009) The social brain: neural basis of social knowledge. Annu Rev Psychol 60:693-716.

Adolphs R (2010) Conceptual challenges and directions for social neuroscience. Neuron 65:752-767.

Ames DL, Jenkins AC, Banaji MR, Mitchell JP (2008) Taking another person's perspective increases self-referential neural processing. Psychol Sci 19:642-644.

Amodio DM, Frith CD (2006) Meeting of minds: the medial frontal cortex and social cognition. Nat Rev Neurosci 7:268-277.

Anderson JR (1998) Social stimuli and social rewards in primate learning and cognition. Behav Processes 42:159-175.

Anderson NH (1968) Likableness ratings of 555 personality-trait words. J Pers Soc Psychol 9:272-279.

Anderson SW, Bechara A, Damasio H, Tranel D, Damasio AR (1999) Impairment of social and moral behavior related to early damage in human prefrontal cortex. Nat Neurosci 2:1032-1037.

Bechara A, Damasio AR, Damasio H, Anderson SW (1994) Insensitivity to future consequences following damage to human prefrontal cortex. Cognition 50:7-15.

Bechara A, Damasio H, Damasio AR (2000) Emotion, decision making and the orbitofrontal cortex. Cereb Cortex 10:295-307.

Bechara A, Damasio H, Damasio AR (2003) Role of the amygdala in decision-making. Ann N Y Acad Sci 985:356-369.

Beckmann M, Johansen-Berg H, Rushworth MF (2009) Connectivity-based parcellation of human cingulate cortex and its relation to functional specialization. J Neurosci 29:1175-1190.

Brainard DH (1997) The Psychophysics Toolbox. Spat Vis 10:433-436.

Buckner RL, Andrews-Hanna JR, Schacter DL (2008) The brain's default network: anatomy, function, and relevance to disease. Ann N Y Acad Sci 1124:1-38.

Bush G, Luu P, Posner MI (2000) Cognitive and emotional influences in anterior cingulate cortex. Trends Cogn Sci 4:215-222.

Cadinu MR, Rothbart M (1996) Self-anchoring and differentiation processes in the minimal group setting. J Pers Soc Psychol 70:661-677.

Carmichael ST, Price JL (1996) Connectional networks within the orbital and medial prefrontal cortex of macaque monkeys. J Comp Neurol 371:179-207.

Dale AM (1999) Optimal experimental design for event-related fMRI. Hum Brain Mapp 8:109-114.

Damasio AR, Tranel D, Damasio H (1990) Individuals with sociopathic behavior caused by frontal damage fail to respond autonomically to social stimuli. Behav Brain Res 41:81-94.

Damasio AR, Tranel D, Damasio H (1991) Somatic markers and the guidance of behavior: theory and preliminary testing. In: Frontal lobe function and dysfunction (Levin HS, Eisenberg HM, Benton AL, eds), pp 217-229. New York: Oxford University.

Damasio AR, Everitt BJ, Bishop D (1996) The Somatic Marker Hypothesis and the possible functions of the prefrontal cortex. Philos Trans R Soc London B 351:1413-1420.

Daw ND, O’Doherty JP, Dayan P, Seymour B, Dolan RJ (2006) Cortical substrates for exploratory decisions in humans. Nature 441:876-879.

Deaner RO, Khera AV, Platt ML (2005) Monkeys pay per view: adaptive valuation of social images by rhesus macaques. Curr Biol 15:543-548.

Dunbar RI, Shultz S (2007) Evolution in the social brain. Science 317:1344-1347.
Eslinger PJ, Damasio AR (1985) Severe disturbance of higher cognition after bilateral frontal lobe ablation: patient EVR. Neurology 35:1731-1741.

Fehr E, Bernhard H, Rockenbach B (2008) Egalitarianism in young children. Nature 454:1079-1083.

Fiske ST, Taylor SE (1984) Social cognition. Reading, MA: Addison-Wesley.

Flavell JH (1999) Cognitive development: children's knowledge about the mind. Annu Rev Psychol 50:21-45.

Friston KJ, Ashburner J, Frith CD, Poline J-B, Heather JD, Frackowiak RSJ (1995) Spatial registration and normalization of images. Hum Brain Mapp 3:165-189.

Glees P, Cole J, Whitty CWM, Cairns H (1950) The effects of lesions in the cingular gyrus and adjacent areas in monkeys. J Neurol Neurosurg Psychiatry 13:178-190.

Gobbini MI, Leibenluft E, Santiago N, Haxby JV (2004) Social and emotional attachment in the neural representation of faces. Neuroimage 22:1628-1635.

Hadland KA, Rushworth MF, Gaffan D, Passingham RE (2003) The effect of cingulate lesions on social behaviour and emotion. Neuropsychologia 41:919-931.

Harris LT, Fiske ST (2007) Social groups that elicit disgust are differentially processed in mPFC. Soc Cogn Affect Neurosci 2:45-51.

Haslam SA, Turner JC, Oakes PJ, McGarty C, Hayes BK (1992) Contextdependent variation in social stereotyping 1: the effects of intergroup relations as mediated by social change and frame of reference. Eur J Soc Psychol 22:3-20.

Heatherton TF, Wyland CL, Macrae CN, Demos KE, Denny BT, Kelley WM (2006) Medial prefrontal activity differentiates self from close others. Soc Cogn Affect Neurosci 1:18-25.

Higgins ET, Bargh JA (1987) Social cognition and social perception. Annu Rev Psychol 38:369-425.

Hornak J, Bramham J, Rolls ET, Morris RG, O’Doherty J, Bullock PR, Polkey CE (2003) Changes in emotion after circumscribed surgical lesions of the orbitofrontal and cingulate cortices. Brain 126:1691-1712.

Hughes C, Leekam S (2004) What are the links between theory of mind and social relations? Review, reflections and new directions for studies of typical and atypical development. Soc Dev 13:590-619.

Kelley WM, Macrae CN, Wyland CL, Caglar S, Inati S, Heatherton TF (2002) Finding the self? An event-related fMRI study. J Cogn Neurosci 14:785-794.

Lou HC, Luber B, Crupain M, Keenan JP, Nowak M, Kjaer TW, Sackeim HA, Lisanby SH (2004) Parietal cortex and representation of the mental self. Proc Natl Acad Sci U S A 101:6827-6832.

Mah LW, Arnold MC, Grafman J (2005) Deficits in social knowledge following damage to ventromedial prefrontal cortex. J Neuropsychiatry Clin Neurosci 17:66-74.

Miezin FM, Maccotta L, Ollinger JM, Petersen SE, Buckner RL (2000) Characterizing the hemodynamic response: effects of presentation rate, sampling procedure, and the possibility of ordering brain activity based on relative timing. Neuroimage 11:735-759.

Mitchell JP (2009) Social psychology as a natural kind. Trends Cogn Sci 13:246-251.

Mitchell JP, Heatherton TF, Macrae CN (2002) Distinct neural systems subserve person and object knowledge. Proc Natl Acad Sci USA 99:15238-15243.

Mitchell JP, Macrae CN, Banaji MR (2006) Dissociable medial prefrontal contributions to judgments of similar and dissimilar others. Neuron 50:655-663.

Mobbs D, Yu R, Meyer M, Passamonti L, Seymour B, Calder AJ, Schweizer S, Frith CD, Dalgleish T (2009) A key role for similarity in vicarious reward. Science 324:900.

Moran JM, Macrae CN, Heatherton TF, Wyland CL, Kelley WM (2006) Neuroanatomical evidence for distinct cognitive and affective components of self. J Cogn Neurosci 18:1586-1594.

Northoff G, Heinzel A, de Greck M, Bermpohl F, Dobrowolny H, Panksepp J (2006) Self-referential processing in our brain: a meta-analysis of imaging studies on the self. Neuroimage 31:440-457.

Ochsner KN, Beer JS, Robertson ER, Cooper JC, Gabrieli JD, Kihsltrom JF, D'Esposito M (2005) The neural correlates of direct and reflected selfknowledge. Neuroimage 28:797-814.

Ojemann JG, Akbudak E, Snyder AZ, McKinstry RC, Raichle ME, Conturo TE (1997) Anatomic localization and quantitative analysis of gradient 
refocused echo-planar fMRI susceptibility artifacts. Neuroimage 6:156-167.

Olsson A, Ochsner KN (2008) The role of social cognition in emotion. Trends Cogn Sci 12:65-71.

Ongür D, Price JL (2000) The organization of networks within orbital and medial prefrontal cortex of rats, monkeys and humans. Cereb Cortex 10:206-219.

Ongür D, Ferry AT, Price JL (2003) Architectonic subdivision of the human orbital and medial prefrontal cortex. J Comp Neurol 460:425-449.

Palomero-Gallagher N, Mohlberg H, Zilles K, Vogt B (2008) Cytology and receptor architecture of human anterior cingulate cortex. J Comp Neurol 508:906-926.

Petrides M, Pandya DN (2007) Efferent association pathways from the rostral prefrontal cortex in the macaque monkey. J Neurosci 27:1157311586.

Platek SM, Kemp SM (2009) Is family special to the brain? An eventrelated fMRI study of familiar, familial, and self-face. Neuropsychologia 47:849-858.

Rendall D, Rodman PS, Emond RE (1996) Vocal recognition of individuals and kin in free-ranging rhesus monkeys. Anim Behav 51:1007-1015.

Rilling JK, Dagenais JE, Goldsmith DR, Glenn AL, Pagnoni G (2008) Social cognitive neural networks during in-group and out-group interactions. Neuroimage 41:1447-1461.

Rolls ET, Hornak J, Wade D, McGrath J (1994) Emotion-related learning in patients with social and emotional changes associated with frontal lobe damage. J Neurol Neurosurg Psychiatry 57:1518-1524.

Rudebeck PH, Buckley MJ, Walton ME, Rushworth MF (2006) A role for the macaque anterior cingulate gyrus in social valuation. Science 313:1310-1312.

Rudebeck PH, Walton ME, Millette BH, Shirley E, Rushworth MF, Bannerman DM (2007) Distinct contributions of frontal areas to emotion and social behaviour in the rat. Eur J Neurosci 26:2315-2326.

Rushworth MF, Buckley MJ, Behrens TE, Walton ME, Bannerman DM (2007) Functional organization of the medial frontal cortex. Curr Opin Neurobiol 17:220-227.
Saleem KS, Kondo H, Price JL (2008) Complementary circuits connecting the orbital and medial prefrontal networks with the temporal, insular, and opercular cortex in the macaque monkey. J Comp Neurol 506:659-693.

Schmitz TW, Kawahara-Baccus TN, Johnson SC (2004) Metacognitive evaluation, self-relevance, and the right prefrontal cortex. Neuroimage 22:941-947.

Schmitz TW, Johnson SC (2006) Self-appraisal decisions evoke dissociated dorsal-ventral aMPFC networks. Neuroimage 30:1050-1058.

Stone VE, Baron-Cohen S, Knight RT (1998) Frontal lobe contributions to theory of mind. J Cogn Neurosci 10:640-656.

Stuss DT (2002) Fractionation and localization of distinct frontal lobe processes: evidence from focal lesions in humans. In: Principles of frontal lobe function (Stuss DT, Knight RT, eds), pp 392-407. New York: Oxford UP.

Tomlin D, Kayali MA, King-Casas B, Anen C, Camerer CF, Quartz SR, Montague PR (2006) Agent-specific responses in the cingulate cortex during economic exchanges. Science 312:1047-1050.

Trivers RL (1971) The evolution of reciprocal altruism. Q Rev Biol 46:35-57.

Vanderwal T, Hunyadi E, Grupe DW, Connors CM, Schultz RT (2008) Self, mother and abstract other: an fMRI study of reflective social processing. Neuroimage 41:1437-1446.

Van Dijk KR, Hedden T, Venkataraman A, Evans KC, Lazar SW, Buckner RL (2010) Intrinsic functional connectivity as a tool for human connectomics: Theory, properties, and optimization. J Neurophysiol 103:297-321.

Van Essen DC (2005) A population-average, landmark-and surface-based (PALS) atlas of human cerebral cortex. Neuroimage 28:635-662.

Volz KG, Kessler T, von Cramon DY (2009) In-group as part of the self: In-group favoritism is mediated by medial prefrontal cortex activation. Soc Neurosci 4:244-260.

Wellman HM, Cross D, Watson J (2001) Meta-analysis of theory-of-mind development: the truth about false belief. Child Dev 72:655-684.

Zhu Y, Zhang L, Fan J, Han S (2007) Neural basis of cultural influence on self-representation. Neuroimage 34:1310-1316. 